\title{
A Circuit Model to a Directive Triangular EBG Antenna
}

\author{
Abdelhak Ferchichi and Ali Gharsallah
}

\begin{abstract}
In This paper, we propose a directive triangular EBG antenna. This approach is derived from using an EBG surface near to a triangular patch with two grilles. The antenna frequency chosen is the $2.45 \mathrm{GHz}$. The directive antenna is simulated by using HFSS and Electrical model. This proposed antenna presents a very high directivity.
\end{abstract}

Keywords-EBG antenna, directive antenna, lumped element, mushroom

\section{INTRODUCTION}

$\mathbf{N}$ OWADAYS, the development of telecommunications systems creates new needs that existing antennas cannot mostly all satisfy. Indeed, they must be broadband, high gain multiband agile diagram, frequency agile, low cost and small. Besides, the antenna is used in all the recent wireless communication systems. The satisfaction of these conditions, as well as towards solutions multifunction antennas require greater adaptability. For all this reasons, the developing of a new structures of antennas consist the challenge of our research laboratory. A structure that is worth considering is the antenna EBG (Electromagnetic Band Gap) because of its potential especially in terms of gain, since it can significantly increase the directivity of a primary source. The EBG antenna is composed of a resonator formed between the ground plane and the material half-EBG (periodic arrangement of dielectric or metallic elements) placed above it. For this reason, EBG structures and their applications in antennas have become a new research field in the RF systems, [1]-[5].

The use EBG antenna was essentially to eliminate some limits in classical antenna such us:

- The suppression of surfaces waves in antenna ground plane.

- Design a simple profile antenna near to ground plane.

- Increase gain and directivity of antenna.

The main problem of this kind of antenna is the complexity of the geometry. This means that the conception step will be very difficult and takes a lot of time. Besides, the absence of analytic model to control all the antenna parameters from the physical parameters of the antenna. On the other hand, due to long simulation time, it will be very difficult to analyze antenna response when we modify some parameters like the excitation, the geometry of metallic element, substrate, ... [6].

In order to simplify the EBG antenna conception step we must use a simple and efficacy simulator. In many works, we use the industrial simulator like HFSS, ADS and CST. All

A. Ferchichi and A. Gharsallah are with Unit of Research Circuits and Electronics Systems High Frequency, Faculty of Science, University El Manar, Tunis, Tunisia (e-mails: abdelhakferchichi@yahoo.fr; ali.gharsallah@gmail.com). those tools are based on numerical methods as the method of moments (MoM), finite element method (FEM), and the finite difference time domain (FDTD) method, which have been well developed over the years. The different methods are based on resolving Maxwell equation on their integral or differential form.

Y. R. Samii presents in [7] the advantages in using EBG structure to improve the performance of microwave and optical systems. In [8], A. R. Weily et all, A. R.Wiekley uses an EBG structure to increase the gain and bandwidth of a resonator antenna; its proposed structure is excitated with a slot technique feed. On the other hand, L. Qiu et all combines the use of an EBG surface with a choke structure to ameliorate the isolation between the emitter and the receiver antenna, [9]. For [10], W. Cao at all develop a novel EBG antenna based on using an EBG structure near to a simple patch; their proposed antenna is characterized by a multi-frequency and dual mode aspect. Lastly, according to [11], M.Kim et all demonstrate that a vertical inductive bridge electromagnetic band gap (VIBEBG) structure can be used as a miniaturization technique of antenna structure dimension, then this last technique is very useful in stop band enhancement and wideband suppression.

We propose in this work, a design of a new directive triangular EBG antenna with a high directivity. In the first section, we described our design of EBG structure where we evaluate EBG parameter by using an electrical model. In the second Section, we developed our proposed directive antenna by placing the chosen EBG design near to a triangular antenna in first structure and then we place two metallic grilles near the EBG triangular patch to increase the directivity. In the last section, we conclude our work and we suggest some perspectives.

\section{The EBG Elementary Cellule}

EBG structures are usually realized by periodic arrangement of dielectric materials or/and metallic conductors. In general, they can be classified to three groups according to their geometric configuration.

The complexity of this type of antenna makes the determination of the size of these structures a very difficult operation. The studies on these antennas have shown that a study of the elementary cell of the metal blocks reflects the behavior of the network blocks, [12]. At this stage, the electrical modeling is effective because from modeling the unit cell, we can determine the geometric dimensions of the metal pad (Fig. 1).

Several studies have examined this type of antennas to offer its equivalent circuit. All these approaches begin by determining the impedance of these surfaces (called High 


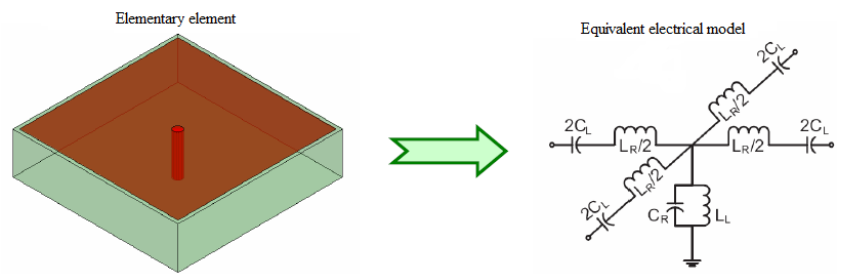

Fig. 1. An electrical model to the EBG cellule.

Impedance Surfaces). From the expression of the impedance, we calculate electrical parameters model. Indeed, the highimpedance surfaces were introduced by D. Sievenpieper et al for the first time in order to remove surface waves patch antenna to increase its performance [13].

In the literature, two models are proposed for the study of SHI: the quasi-static and D. F. Sievenpieper introduced by the dynamic model introduced by Simovski and all, [14]. For both cases, the approximation of the expression level of the impedance changing.

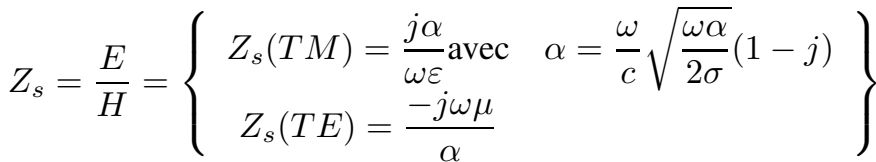

D. F. Siervenpiper Expression

$$
Z_{s}=\frac{\frac{j}{\sqrt{\varepsilon_{r}}} \tan \left(k \sqrt{\varepsilon_{r}} h\right)}{1-\frac{k a\left(1+\varepsilon_{r}\right)}{\pi \sqrt{\varepsilon_{r}}} \log \left(\frac{2 a}{\pi g}\right) \tan \left(k \sqrt{\varepsilon_{r}} h\right)}
$$

C. R. Simovski Expression

Based on the error in the accuracy of the resonant frequency, the model D. Sievenpieper which is most effective with an error rate of $10 \%$. For this reason, we have adopted the model of D. Sievenpieper to calculate the parameters of the electrical model. Thus, $L_{R}$ and $C_{L}$ can be calculated using equations:

$$
\begin{gathered}
L_{R}=\mu_{0} \mu_{r} h \\
C_{L}=\frac{b \varepsilon_{0}\left(1+\varepsilon_{r}\right)}{\pi} A \cosh \left(\frac{a}{g}\right)
\end{gathered}
$$

With:

$h$ : thickness of substrate,

$b$ : width of metallic element,

$A$ : distance between the metallic elements, $g$ : width gap.

\section{The Proposed EBG AntennA}

Due to the advantages of creating an EBG surfaces, we use it near to a simple antenna in order to see the modification antenna parameter.

\section{A. Geometry}

In our case, we have chosen to create an EBG surface near to a rectangular patch. First we place the via hole in the center of each metallic patch then we use a coaxial excitation to our planar antenna. This circuit is mounted in a planar structure with a substrate and a ground plane. The whole structure is put into a box. The substrate we used is FR4 epoxy with a permittivity $\varepsilon_{r}=4.4$ and a height $h=1.6 \mathrm{~mm}$. The two designs are shown in Fig. 2.

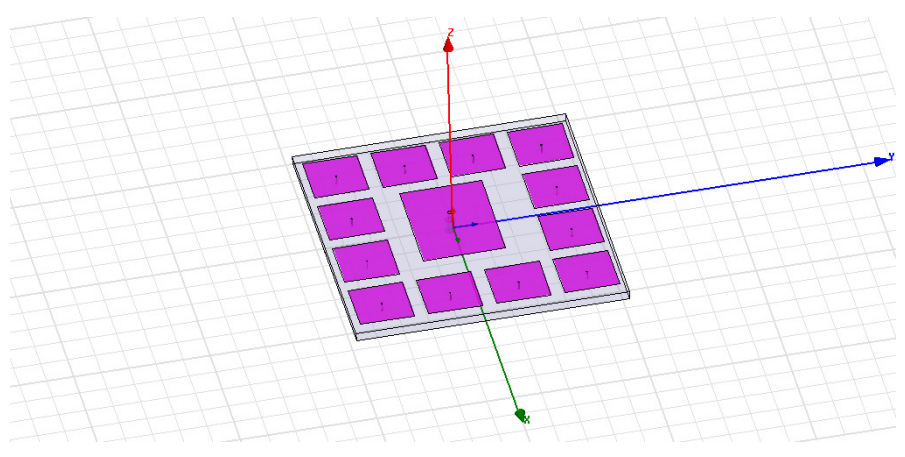

Fig. 2. The geometry of the two designs of EBG structure.

\section{B. The Proposed Electrical Model for the EBG Antenna}

EBG structures (Electromagnetic Band Gap) have many advantages and are currently used in several applications. Among these applications, we use these structures for antennas with high gain and directivity. In our work, we thought to use these forms as antennas for RFID reader. The use of these structures is not clear due to the lack of analytical equations for controlling the parameters of the antenna according to the geometry of the antenna. Indeed, the geometry of the antenna is very complex for we are obliged to determine several parameters such as the geometrical dimensions of the patch, the geometrical dimensions of the metal pad, the spacing between the metal and stone setting optimal characteristics the via hole: diameter and position.

Thus, the electric model then becomes an indispensable tool that allows us to find a direct relationship between the resonance frequency of an EBG structure and all these geometric parameters from the electric model. The latter is constructed in the following steps:

- Replace each elementary metallic cellule by an electric model (Fig. 1).

- Modeling the coupling between the metal blocks by a coupling capacitance as explaining in our previous works, [15].

- Replace the patch antenna excited by a coaxial cable by its RLC electrical model (Fig. 3). The parameters model can be calculated using the equation developed in our previous works, [16], [17].

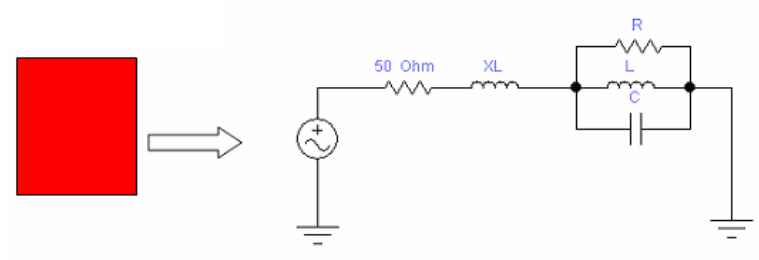

Fig. 3. An electrical model to rectangular patch. 
- Modeling the coupling between the patch and the surrounding pavement by a coupling capacitance.

To validate our model, we took an EBG structure consisting of four rows of metal blocks surrounding a patch antenna as an antenna Mushroom. The proposed electrical model is shown in Fig. 4.

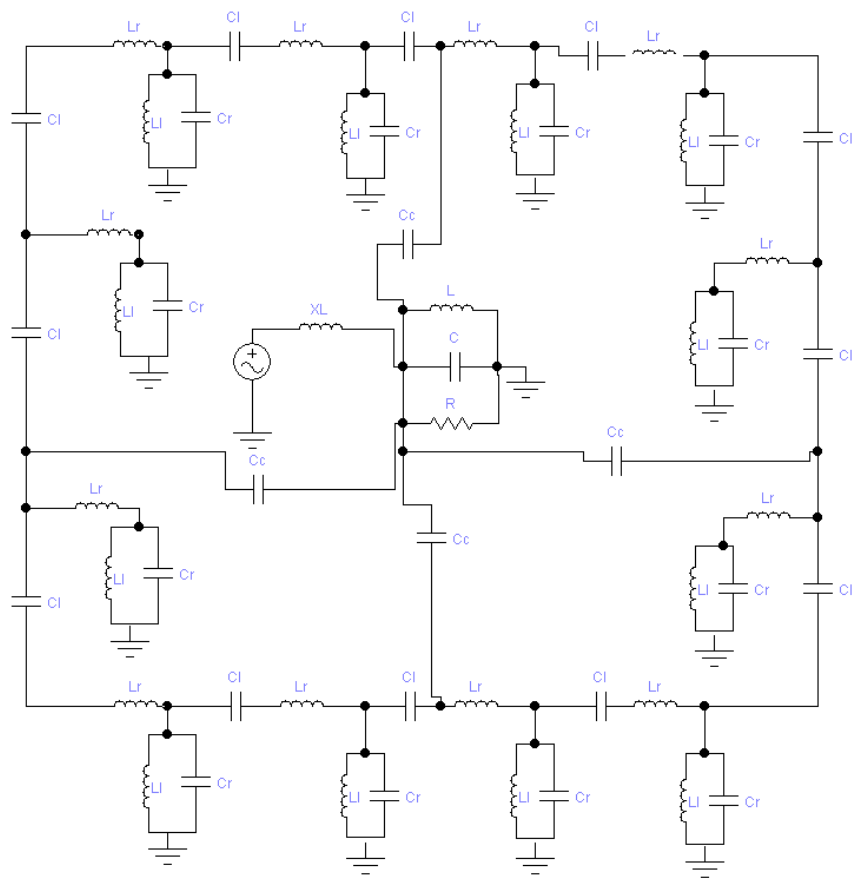

Fig. 4. The electrical model of proposed EBG structure.

\section{Result of Simualation}

For this type of structures, the simulation takes a long time especially if you take a large number of metal blocks. The increase in metal blocks makes the simulation very slow HFSS and may take several hours! In our case, the simulation in HFSS rectangular patch with four rows of stones is hard metal 38 minutes. The simulation model electric gave us results in less than a second.

Simulation results are shown in Fig. 5.

In order to compare the obtained result by using each tool: HFSS and electrical model, we dress Tab. I.

TABLE I

COMPARISON BETWEEN EleCtrical MOdEL RESUlTS AND HFSS RESULTS OF PROPOSED ANTENNA

\begin{tabular}{lll}
\hline \hline Antenna & HFSS & Electrical Model \\
Resonant frequency $(\mathrm{GHz})$ & 2.45 & 2.45 \\
BW $(\mathrm{MHz})$ & 100 & 50 \\
Simulation Time & 38 minute & 1.5 seconds \\
\hline \hline
\end{tabular}

As can be seen in the table, the proposed model gives us the same resonant frequency of HFSS in a very short time. Thus, we can use the proposed model in an analytic study when modifying one of geometrical antenna parameter. Once the needed resonant frequency is obtained; we can also calculate

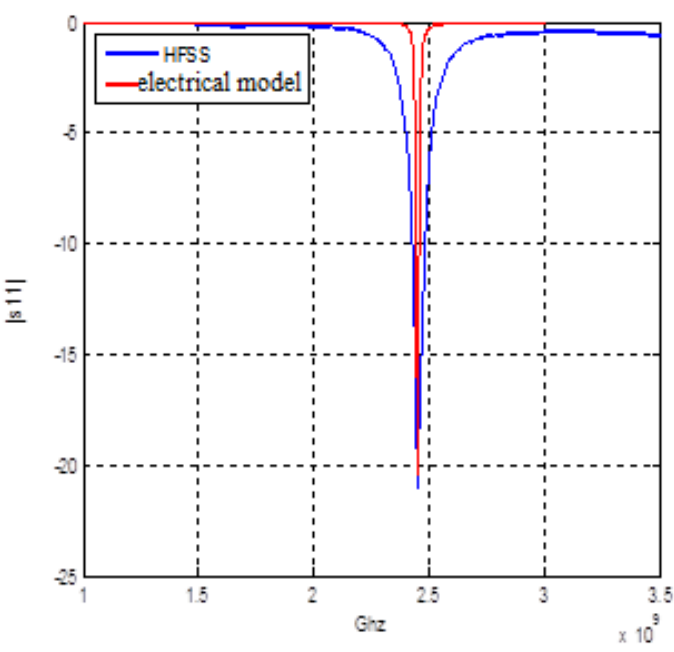

Fig. 5. Return loss of the proposed EBG structure.

the physical parameter of the antenna from the lumped element which seems very interesting. We note also, that the proposed model has a narrow band width comparing to real patch due to differences between the real losses and those calculated by the circuit model.

\section{The Proposed Directive Triangular EBG ANTENNA}

In this last section, we tried to combine two techniques in order to propose a directive EBG antenna by placing two grilled surfaces near to EBG structure. First, we begin by choosing the best EBG surface. Then, we design a simple EBG triangular patch. Finally, we design the proposed design by introducing the two metallic grilles.

\section{A. EBG Surface}

In our case, we have chosen to do a comparison between the EBG parameter when we changing the via hole position. First we place the via hole in the center of the metallic patch then we put it in the middle of one side of the square patch. This circuit is mounted in a planar structure with a substrate and a ground plane. The whole structure is put into a box. The substrate we used is FR4 epoxy with a permittivity $\varepsilon_{r}=4.4$ and a height $h=1.6 \mathrm{~mm}$. The two designs are shown in Fig. 6 .

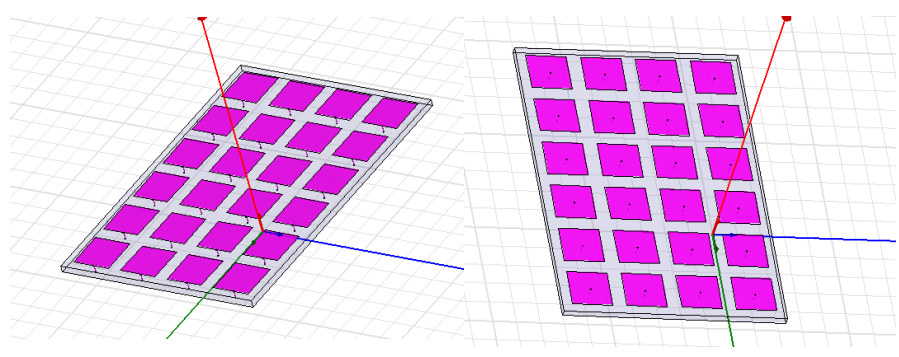

Fig. 6. The two EBG surface.

According to the simulation results (Fig. 7), we note that the two Mushroom structures present two different bands Gap 

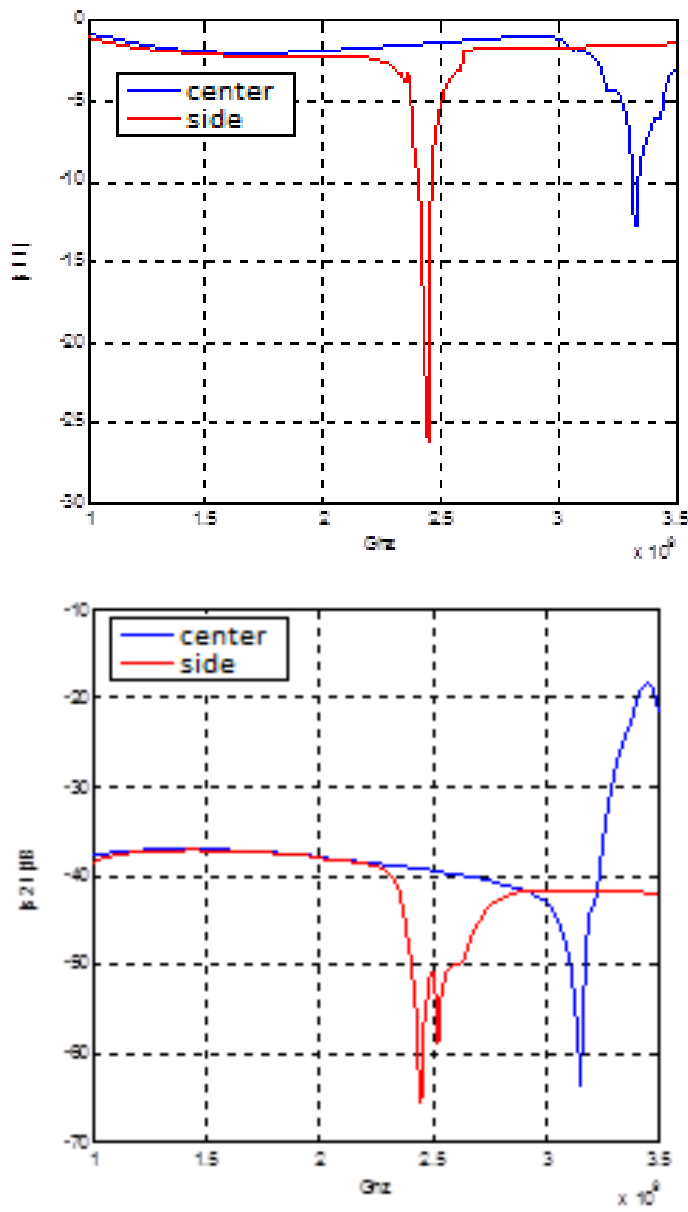

Fig. 7. Simulation results of the two configuration of EBG structure.

widths. Indeed, the position of the via hole has a major effect on the characteristics of these surfaces. In our case, it is clear that the use of the structure with via the middle of one side is more effective since it has some advantages, which include:

- According to the reflection coefficient S11, the resonance frequency is about $2.45 \mathrm{GHz}$ which is a frequency RFID.

- According to the transmission coefficient S21, the structure has a band gap between $2.25 \mathrm{GHz}$ and $2.8 \mathrm{GHz}$.

As the frequency of work of the proposed antenna $(2.45 \mathrm{GHz})$ is in the band gap structure [2.25GHz, $2.8 \mathrm{GHz}$, a direct influence on the radiation parameters of our antenna is obtained.

\section{B. First EBG Triangular Structure}

To take advantage of Mushroom structures, it was thought to simulate a triangular antenna in a Mushroom (Fig. 8).

The simulation of the proposed EBG antenna, gave us the reflection coefficient S11 shown in Fig. 5. We note that our antenna has a resonant frequency equal to $2.45 \mathrm{GHz}$ and a bandwidth of about $90 \mathrm{MHz}$. Besides, the proposed antenna has a very high gain equal to $14.9 \mathrm{~dB}$ (Fig. 9).

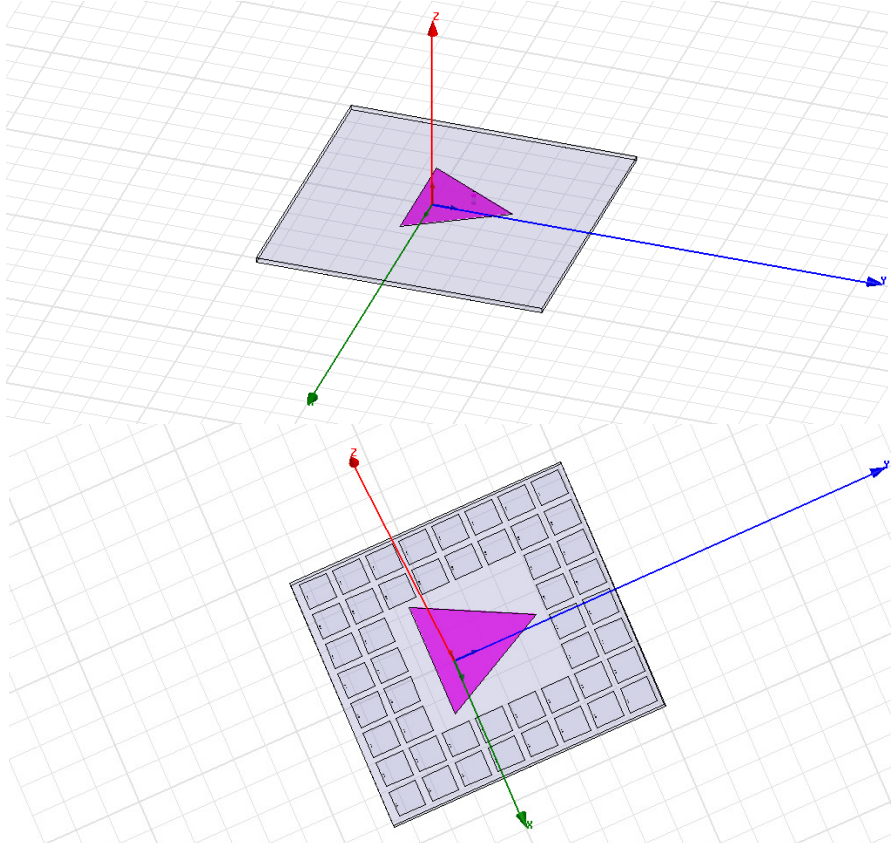

Fig. 8. The triangular EBG antenna.
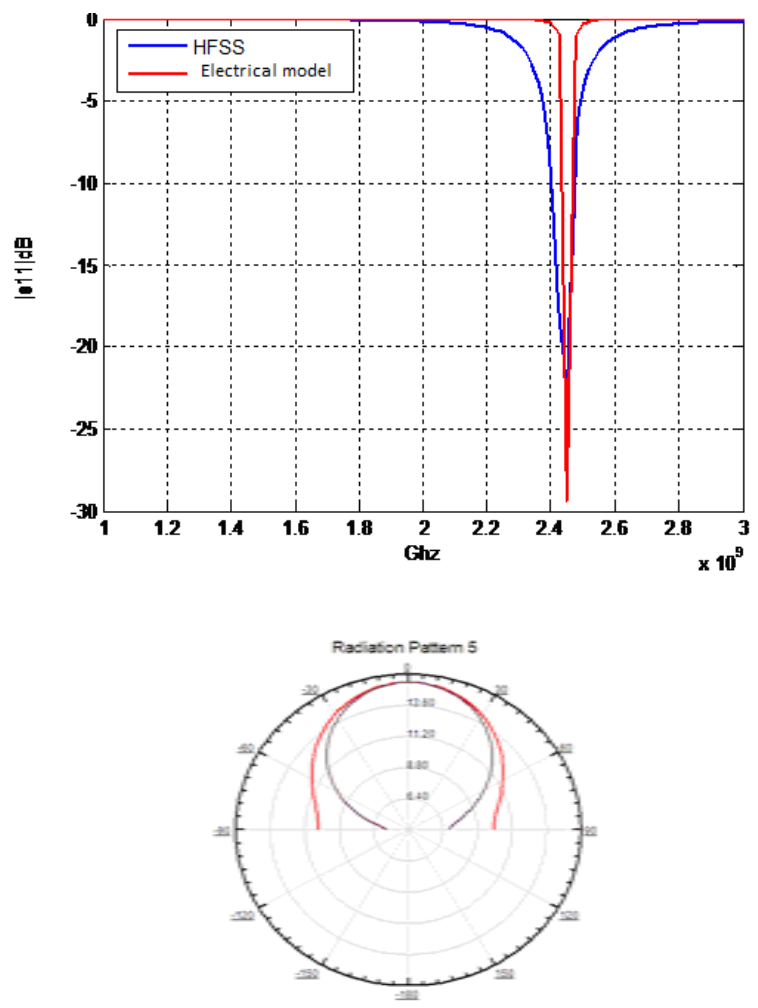

Fig. 9. Simulation result of EBG triangular patch.

\section{The Proposed Grilled EBG Traiangular Antenna}

The novel geometry is presented in Fig. 10. We used the same substrate as above but we change only the rectangular by a triangular antenna. 


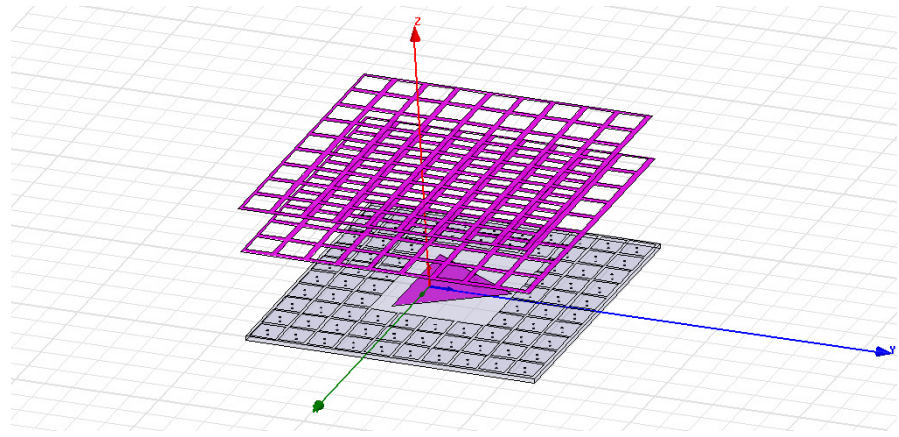

Fig. 10. The proposed grilled triangular EBG antenna.

\section{Result of Simulation}

The simulation of the proposed EBG antenna, gave us the reflection coefficient S11 shown in Fig. 11. We note that our antenna has a resonant frequency equal to $2.45 \mathrm{GHz}$ and a bandwidth of about $100 \mathrm{MHz}$. Besides, the proposed antenna has a very high directivity equal to $24.84 \mathrm{~dB}$.

a)

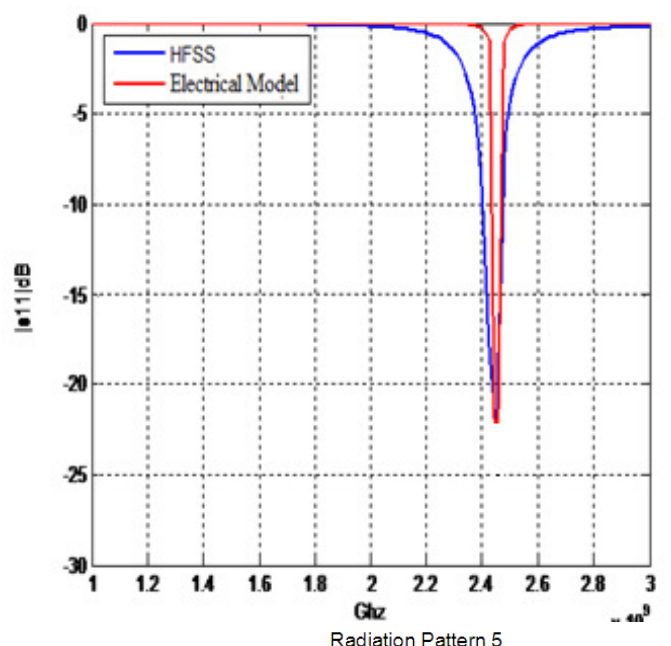

b)

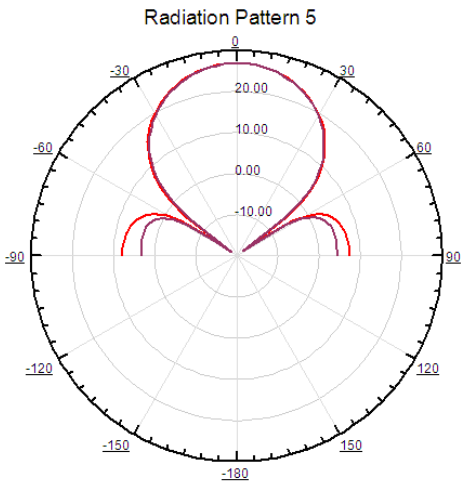

Fig. 11. Simulation result of grilled triangular EBG antenna. a) return loss b) directivity.

As figured in Tab. II, we note that both the antenna simulated with HFSS and the electrical model has the same resonant frequency and a comparable band width. But when, seeing the simulation time, we can say that the electrical model is very fast which can be useful in antenna design step.
TABLE II

COMPARISON BETWEen EleCtRICAL MOdEl RESUltS AND HFSS RESUlts of Directive TRIANGUlar EBG ANTENNA

\begin{tabular}{lll}
\hline \hline Antenna & HFSS & Electrical Model \\
Resonant frequency $(\mathrm{GHz})$ & 2.45 & 2.45 \\
BW (MHz) & 100 & 70 \\
Simulation Time & $3 \mathrm{~h} .24 \mathrm{~min}$ & 1.5 second \\
\hline \hline
\end{tabular}

\section{CONCLUSION}

A new directive EBG antenna was designed and evaluated. The proposed antennas have a resonant frequency equal to $2.45 \mathrm{GHz}$ and it presents a very high directivity which can ameliorate the performance of RF systems. The circuit model based on simple lumped element was a very useful and fast tool to simplify the design, the simulation and the control of the entire parameter antenna.

\section{REFERENCES}

[1] F. Yang and Y. Rahmat-Samii, Electromagnetic Band Gap structure in Antenna Engineering. Cambridge University Press, 2009.

[2] — "Microstrip antennas integrated with electromagnetic band-gap (EBG) structures: A low mutual coupling design for array applications," IEEE Transactions on Antennas Propagation, vol. 51, no. 10, pp. 29362946, October 2003.

[3] A. E. I. Lamminen, A. R. Vimpari, and J. Säily, "UC-EBG on LTCC for $60-\mathrm{GHz}$ frequency band antenna applications," IEEE Transactions on Antennas Propagation, vol. 57, no. 10, pp. 2904-2912, October 2009.

[4] R. Baggen, M. Martinez-Vazquez, J. Leiss, S. Holzwarth, L. S. Drioli, and P. deMaagt, "Low profile Galileo antenna using EBG technology," IEEE Transactions on Antennas Propagation, vol. 56, no. 3, pp. 667674, March 2008.

[5] L. Li, X. J. Dang, B. Li, and C. H. Liang, "Analysis and design of waveguide slot antenna array integrated with electromagnetic band-gap structures," IEEE Antennas Wireless Progagation Letters, vol. 5, pp. 111-115, 2006.

[6] Q. R. Zheng, Y. Q. Fu, and N. C.Yuan, "A novel compact spiral electromagnetic band-gap (EBG) structure," IEEE Transactions on Antennas Propagation, vol. 56, no. 6, pp. 1656-1660, June 2008.

[7] Y. Rahmat-Samii, "The Marvels of Electromagnetic Band Gap (EBG) Structures," Applied Computational Electromagnetics Society Journal, vol. 18, no. 3, pp. 1-10, 2003.

[8] A. R. Weily, K. P. Esselle, T. S. Bird, and B. C. Sanders, "Dual resonator 1-D EBG antenna with slot array feed for improved radiation bandwidth," IET Microwaves, Antennas and Propagation, vol. 1, no. 1, pp. 198-203, 2007.

[9] E. L. Qiu, F. Zhao, K. Xiao, S.-L. Chai, and J.-J. Mao, "TransmitReceive Isolation Improvement of Antenna Arrays by Using EBG Structures," IEEE Antennas and Wireless Propagation Letters, vol. 11, pp. 93-96, 2012.

[10] E. L. W. Cao, B. Zhang, A. Liu, T. Yu, D. Guo, and X. Pan, "MultiFrequency and Dual-Mode Patch Antenna Based on Electromagnetic Band-gap (EBG) Structure," IEEE Antennas and Propagation, vol. 60, no. 12, pp. 6007-6012, December 2012.

[11] M. Kim, K. Koo, J. Kim, and J. Kim, "Vertical Inductive Bridge EBG (VIB-EBG) Structure With Size Reduction and Stopband Enhancement for Wideband SSN Suppression," IEEE Antennas and Wireless Propagation Letters, vol. 22, pp. 403-405, 2012.

[12] L. Li, et al, "Locally Resonant Cavity Cell Model for Electromagnetic Band Gap Structures," IEEE Transactions On Antennas And Propagation, vol. 54, no. 1, January 2006

[13] D. Sievenpiper, et all, "High-impedance surface electromagnetic surfaces with a forbidden frequency band," IEEE Transactions on Microwave Theory and Techniques, vol. 47, no. 11, pp. 2059-2074, November 1999.

[14] C. R. Simovski and A. A. Sochava, "High-impedance surfaces based on self resonant grids. analytical modeling and numerical simulations," Progress In Electromagnetics Research, pp. 239-256, 2003.

[15] A. Ferchichi, N. Fadlammah, and A. Gharsallah, "Novel electrical model to an antenna array," in 27th Annual Review of Progress in Applied Computational Electromagnetics ACES, USA, Williamsburg, Virginia, 27-31 March 2011, pp. 689-694. 
[16] A. Ferchichi, N. Fadlallah, N. Sboui, and A. Gharsallah, "Analysis and design of Printed Fractal Antenna by Using an Adequate Electrical Model," International Journal of Communication Networks and Information Security IJCNIS, vol. 1, no. 3, December 2009.
[17] A. Ferchichi and A. Gharsallah, "Analysis of a square parasitic antenna by using lumped element," International Journal of electronics and telecommunication, vol. 58, no. 3, September 2012. 